OPEN ACCESS

Edited by:

Serge Ankri,

Technion-Israel Institute of

Technology, Israel

Reviewed by:

Jaishree Paul,

Jawaharlal Nehru University, India

Stefan Kappe,

Center for Infectious Disease

Research, United States

*Correspondence:

Cecilia Ximénez

cximenez@unam.mx

Specialty section: This article was submitted to

Parasite and Host,

a section of the journal Frontiers in Cellular and Infection

Microbiology

Received: 23 March 2018 Accepted: 19 September 2018

Published: 18 October 2018

Citation:

Gonzalez Rivas E, Ximenez C Nieves-Ramirez ME, Moran Silva P, Partida-Rodríguez O, Hernandez EH, Rojas Velázquez L, Serrano Vázquez $A$

and Magaña Nuñez U (2018)

Entamoeba histolytica Calreticulin Induces the Expression of Cytokines in

Peripheral Blood Mononuclear Cells Isolated From Patients With Amebic Liver Abscess.

Front. Cell. Infect. Microbiol. 8:358. doi: 10.3389/fcimb.2018.00358

\section{Entamoeba histolytica Calreticulin Induces the Expression of Cytokines in Peripheral Blood Mononuclear Cells Isolated From Patients With Amebic Liver Abscess}

\author{
Enrique Gonzalez Rivas, Cecilia Ximenez*, Miriam Enriqueta Nieves-Ramirez, \\ Patricia Moran Silva, Oswaldo Partida-Rodríguez, Eric Hernandez Hernandez, \\ Liliana Rojas Velázquez, Angelica Serrano Vázquez and Ulises Magaña Nuñez \\ Laboratorio de Inmunología, Unidad de Investigación de Medicina Experimental, Facultad de Medicina, Universidad Nacional \\ Autónoma de México (UNAM), Ciudad de Mexico, Mexico
}

Calreticulin (CRT) is a highly conserved protein in the endoplasmic reticulum that plays important roles in the regulation of key cellular functions. Little is known about the participation of E. histolytica CRT (EhCRT) in the processes of pathogenicity or in the modulation of the host immune response. The aim of this study was to evaluate the role of CRT in the proliferation and the cytokine profile in peripheral blood mononuclear cells (PBMCs) from patients with amebic liver abscess (ALA) during the acute phase (AP-ALA) of the disease compared to patients during the resolution phase (R-ALA). The PBMCs from each participant were cocultured with EhCRT and tested by the colorimetric method to evaluate their proliferation index (PI). The supernatants were subjected to an enzyme-linked immunosorbent assay (ELISA) to evaluate the concentration of cytokines. The mean values of all groups were compared using the independent $t$-test. When the Pls of individuals without diagnosis of liver abscess (NEG) were compared, there were no statistically significant differences in the proliferation of PBMCs between patients with AP-ALA and R-ALA when stimulated with EhCRT or concanavalin A (ConA). However, the levels of interleukins [IL-6, IL-10, granulocyte colony stimulating factor (GCSF), and transforming growth factor $\beta 1$ (TGF $\beta 1$ )] were higher in patients with AP-ALA, whereas in patients with R-ALA, higher levels of interferon gamma (IFN $\gamma$ ) were detected. These results suggest that EhCRT acts as a mitogen very similar to the activity of ConA. In addition, EhCRT is an excellent immunogen for the specific activation of PBMCs, inducing the differential expression of ILs depending on the outcome of disease, determining the type of immune response: a Th2 cytokine profile during the acute phase and a Th1 profile during the resolution phase.

Keywords: amebic liver abscess, calreticulin, Entamoeba histolytica, mitogen, proliferation index, interleukins 


\section{INTRODUCTION}

Infection with the enteric protozoan E. histolytica is one of the leading causes of death worldwide. The disease is a consequence of the parasite's abilities to invade the colon, causing amebic colitis. E. histolytica can disseminate to the liver via the portal venous system, resulting in amebic liver abscess (ALA). However, approximately $90 \%$ of infected people are asymptomatic cyst carriers (Haque et al., 2003). The molecular mechanisms by which this parasite causes invasive amebiasis are not fully understood. E. histolytica has adherence and cytotoxicity factors that are essential for its survival, but they are not directly responsible for ALA formation. It is known that the limitation and prevention of recurrent invasive amebiasis requires the development of an effective immune response. Thus, it is likely that the acute inflammatory response associated with E. histolytica infection is a key factor for the development of ALA (Chadee et al., 1985).

Parasite-specific immune responses are regulated by cytokines and chemokines that lead to the development of immunity, but these responses also contribute to infection, inducing pathogenesis and parasite persistence (Talvani et al., 2004). Little is known regarding the amebic signals that initiate an acute inflammatory response.

It has been reported that in mice infected with E. histolytica, host tissue damage is attributed primarily to the lectin activity of the galactose/N-acetyl-D-galactosamine (Gal/GalNAc) from E. histolytica, which promotes the accumulation of mononuclear cells, including neutrophils, inflammatory monocytes, and macrophages, at the site of infection (Blazquez et al., 2007). Intestinal epithelial cells infected with E. histolytica in vitro produce elevated levels of the cytokines, interleukin-8 (IL-8), growth-regulated protein alpha (GRO- $\alpha$ ), granulocyte macrophage colony-stimulating factor (GMCSF), and IL-1 (Eckmann et al., 1995). Treatment of cultured human intestinal cells with the lectin Gal/GalNAc from pathogenic and nonpathogenic entamebas (E. histolytica and $E$. dispar) results in the secretion of chemoattractant and proinflammatory cytokines (Sharma et al., 2005), suggesting that these cells and cytokines also contribute to tissue damage, participating in the mechanisms of initiation, amplification, or limitation of the inflammatory processes during invasive amebiasis.

The identification of the mediators involved in leukocyte activation during infection by E. histolytica is of fundamental importance for understanding host responses in amebiasis. Cellular interactions and cytokines have been reported during amebic infections, and cytokines have been shown to be able to regulate monocyte function and increase the amebicidal activity of monocytes (Seydel et al., 2000; Lotter et al., 2013).

It is still not clear what other elements in the dynamics of host-parasite relationship define the outcome of infection, especially regarding the regulation of the immune response against $E$. histolytica.

To obtain further evidence about the relationships of immune cells with $E$. histolytica, other proteins have been studied to investigate the intracellular signals that promote the host immune response. Some examples include the cysteine proteinases 1 and 5 ( $\mathrm{CP} 1$ and $\mathrm{CP} 5$ ) that breakdown IgA1 and IgA2 antibodies. These proteins cleave the Fc region that interacts with parasite surface receptors and mediates effector functions that can mask immunogenic surface molecules with inert Fab fragments, thus helping to prevent the parasite expulsion from the intestinal lumen (Garcia-Nieto et al., 2008).

The role of calreticulin (CRT) in host-parasite interactions has recently become a major area of research. The CRT genes from many parasites (Trypanosoma, Leishmania, Entamoeba, Onchocerca, Schistosoma, and Haemonchus) have been cloned and sequenced (Rokeach et al., 1994; Joshi et al., 1996; El-Gengehi et al., 2000; Marcelain et al., 2000; González et al., 2002; Suchitra and Joshi, 2005).

Although the functions of CRT are conserved in vertebrates, some CRT functions differ among parasites (Nakashi et al., 1998; Ferreira et al., 2004); parasite CRTs bind host C1q and inhibit Clq-dependent complement activation. The CRT of Haemonchus contortus binds host C-reactive protein and C1q (Naresha et al., 2009). The ecto-parasite Amblyomma americanum secretes CRT during feeding, suggesting that the anticoagulant ability of CRT may prevent blood clotting and allows the parasite to feed on the host and induce host antiparasite responses (Jaworski et al., 1995). The presence of CRT in the penetration gland cells of Schistosoma suggests that this molecule may be important for the host skin penetration (Khalife et al., 1994).

Previously, we reported the presence of CRT in E. histolytica $(E h C R T)$ and that this protein induces an important immunogenic response in the human host. More than $90 \%$ of patients with ALA develop high levels of serum antibodies against EhCRT (González et al., 2002). We also reported the cloning of the CRT gene from E. histolytica, and the preparation of mono-specific antibodies against recombinant CRT. The immunohistochemical assays on trophozoites show that EhCRT is in cytoplasmic vesicles and in vesicles that are in close contact with the inner cytoplasmic membrane (González et al., 2011). In addition, it was demonstrated that the CRT from both pathogenic E. histolytica and nonpathogenic E. dispar species specifically interact with human $\mathrm{C} 1 \mathrm{q}$ molecules and inhibit the activation of the classical complement pathway (Ximénez et al., 2014). This activity is consistent with that reported by Vaithilingam et al. (2012). The trophozoites activated by the presence of jurkat cells clearly show the binding of C1q to CRT on the surface of the phagocytic mouths during the process of erythrophagocytosis.

However, the activation of the host immune response and the cytokine profile induced by EhCRT have not yet been investigated.

In this study, we analyzed the proliferation and cytokine production of peripheral blood mononuclear cells (PBMCs) cultures isolated from ALA patients during the acute phase of the disease and from individuals who resolved ALA in order to characterize the cytokines profiles produced in response to EhCRT. 


\section{METHODS}

\section{Ethics Statement}

The present work was designed according to the guidelines for the management of human samples for experimental purposes as indicated in the Official Regulation NOM-12SSA3-2007 included in the General Health Law of Mexican Health Ministry. In addition, the project was approved by the Scientific and Ethics Committee of the Faculty of Medicine from the National Autonomous University of Mexico. Patients were informed about the purposes of the project, the sampling, and the potential risks during procedure, and the patients were invited to voluntarily participate by signing an informed consent letter.

\section{Study Groups and Biological Samples}

Patients with diagnosis of ALA admitted to the Internal Medicine, Gastroenterology, and Infectiology Services of the General Hospital of Mexico Dr. Eduaro Liciaga were recruited. These patients formed the group with acute phase amebic liver abscess (AP-ALA). The patients in the resolution phase of ALA (R-ALA) were recruited from a search of the archives of discharged patients from the General Hospital of Mexico, and they formed the ALA resolution group (R-ALA). Three fecal samples were collected from each patient from both groups at 1-week intervals after their hospitalization for microscopic examination for parasites. At the time of collection of the third sample, $10 \mathrm{ml}$ of blood was drawn to evaluate serum antibodies against E. histolytica by enzyme-linked immunosorbent assay (ELISA), using an $\mathrm{OD}_{490 \mathrm{~nm}} \geq 0.5$ as an indicator of a positive result (Morán et al., 2007). The remaining sample was used to isolate PBMCs.

A third group, named the negative control (NEG), was formed with clinically healthy individuals from the Blood Bank of the General Hospital of Mexico, with ELISA serum levels of antiamebic antibodies below the OD threshold $(\mathrm{OD}<0.5)$. Using the same protocol for ALA groups, three fecal samples and 10$\mathrm{ml}$ blood samples were taken for microscopic examination and PBMC isolation, respectively. Ten individuals for the group were included.

\section{Isolation and Culture of PBMC}

The PBMCs were isolated from $10 \mathrm{ml}$ peripheral blood samples collected from each participant in tubes with K2 ethylenediaminetetraacetic acid (EDTA) as anticoagulant (BD Vacutainer, Ref 368171). Cells were separated on a FicollHypaque gradient (Gibco, Life Technologies, Grand Island, NY, USA), and the PBMC pellet was separated and washed three times with phosphate-buffered saline PBS. After separation, PBMCs were centrifuged and resuspended in Roswell Park Memorial Institute medium RPMI culture medium supplemented with $10 \%$ fetal bovine serum. The cells $\left(1 \times 10^{6}\right.$ cells $\left./ \mathrm{ml}\right)$ were incubated with $\mathrm{rEhCRT}(5 \mu \mathrm{g} / \mathrm{ml})$ at $37^{\circ} \mathrm{C}$ with $5 \% \mathrm{CO}_{2}$; concanavalin A (ConA) (Sigma Chemical St. Louis, MO USA) was used as a stimulating factor at $10 \mu \mathrm{g} / \mathrm{ml}$ for different periods of time (24, 48,72 , and $96 \mathrm{~h}$ ). Each experiment was performed in duplicate. At each time point, the cultures were centrifuged for $10 \mathrm{~min}$ at $1,000 \times \mathrm{g}$, and the supernatant was reserved for cytokine analysis.

\section{Recombinant EhCRT Production}

Full-length rEhCRT protein was expressed and purified as previously described (González et al., 2011). Briefly, the plasmid pBluescript-KS+ (pbKS+) was used to clone and express the 1,178 bp Ehcrt gene (GB-EAL649855.1) (Loftus et al., 2005), which produces the full-length protein; it was subcloned into the prokaryotic expression vector pProEX HT-b (Gibco Life Technologies) to express the CRT protein with a six-histidine tag at the N-terminal end. Competent Escherichia coli BL21 cells were transformed with the recombinant plasmid. The expression of recombinant protein $\mathrm{rEhCRT}$ was induced with a final concentration of $1 \mathrm{mM}$ isopropyl- $\beta$-D-thiogalactoside. The QIAexpressionist system (Qiagen, Valencia, CA, USA) was used to purify the recombinant protein, and, briefly, the cells were harvested by centrifugation at 3,000 $\times \mathrm{g}$ for $12 \mathrm{~min}$, and the bacterial pellet was resuspended in $5 \mathrm{ml}$ lysis buffer $(8 \mathrm{M}$ urea, $0.1 \mathrm{M} \mathrm{NaH}_{2} \mathrm{PO}_{4}$, and $0.1 \mathrm{M}$ Tris- $\mathrm{HCl}, \mathrm{pH}$ 8.0). The lysate was added to a $50 \%$ suspension of Ni-NTA agarose (Qiagen,). The mixture was passed through a filtration column, and the recombinant protein was eluted with 8 M-urea buffer $\mathrm{pH}$ 4.5. The selected fractions were dialyzed against $19 \mathrm{mM}$ PBS. Protein concentration was determined by the Bradford method, and the quality was evaluated by $12 \%$ sodium dodecyl sulfate polyacrylamide gel electrophoresis (SDS-PAGE). The reactivity grade of the recombinant protein was tested against sera from patients with ALA and for anti-E. coli lipopolysaccharide antibody (LPS) antibody (ab211144, Abcam) by Western blot.

\section{Proliferation Index (PI)}

To obtain the PI at 24, 48, 72, and $96 \mathrm{~h}, 20 \mu \mathrm{l}$ of $5 \mu \mathrm{g} / \mathrm{ml}[3-(4,5-$ dimethylthiazol-2-yl)-2,5-diphenyltetrazolium bromide] (MTT) (Sigma) was added to each well containing PBMCs incubated with $\mathrm{rEhCRT}$ or ConA and incubated at $37^{\circ} \mathrm{C}$ for $1 \mathrm{~h}$ in a humidified incubator with $5 \% \mathrm{CO}_{2}$. After incubation, the plates were centrifuged at $1,000 \times \mathrm{g}$ for $10 \mathrm{~min}$ and the supernatant was discarded. The cells were resuspended in $300 \mu \mathrm{l}$ dimethyl sulfoxide (Sigma) and the optical density (OD) was measured at $570 \mathrm{~nm}$ in an ELISA plate reader (EL $\times 800$ BioTek). The proliferation index was calculated by [OD of test sample-OD of negative control/OD of negative control]. (Verma et al., 2010)

\section{Cytokine Detection by ELISA}

The supernatants of the PBMC cultures, treated with rEhCRT, ConA, or without stimulus (RPMI-10\% SFB), were tested for detection of interleukins IL-2, IL-4, IL-5, IL-6, IL-10, IL12, IL-13, IL-17A, interferon gamma (IFN $\gamma$ ), tumor necrosis factor alpha $(\mathrm{TNF} \alpha)$, granulocyte colony stimulating factor (GCSF), and transforming growth factor betal (TGF $\beta 1$ ) using a multianalytic ELISA array kit (MEH-003A, Qiagen), according to manufacturer's instructions. The concentrations of the cytokines are given in $\mathrm{pg} / \mathrm{ml}$ and were calculated using the standard curve provided in the kit.

\section{Statistical Analysis}

All values are expressed as the means \pm S.D. The student's $t$ test for unpaired results was used for the evaluation of differences 
TABLE 1 | Demographic characteristics of individuals in the AP-ALA, R-ALA, and healthy control (NEG) groups.

\begin{tabular}{lccccc}
\hline Groups & Age (range in years) & Male & Female & Total & ELISA (OD) \\
\hline AP-ALA & 39.11 & 9 & 1 & 10 & $0.87 \pm 0.043$ \\
R-ALA & 39.72 & 6 & 4 & 10 & $1.0 \pm 0.025$ \\
NEG & 47.62 & 7 & 3 & 10 & $0.15 \pm 0.024$
\end{tabular}

The antibodies against E. histolytica antigens present in all belonging to different studied groups were evaluated by ELISA. The data are expressed as the average $O D \pm S D$ in each group.

between cytokine concentrations in each group. Differences were statistically significant when $P \leq 0.05$.

\section{RESULTS}

\section{Study Participants and E. histolytica Antibody Concentration}

The demographic features of each group are shown in Table 1. Among all the individuals, the mean age was $39 \pm 7$ years old, and $70 \%$ were male and $30 \%$ were female. The microscopic examinations were negative in all samples. Results of ELISA assay are shown as OD $490 \mathrm{~nm}$ values, considering negative results when values were under the cut value of 0.520 . The mean value for the negative group was 0.15, the AP-ALA group was 0.87 , and the R-ALA group was 1.0.

\section{Purification of rEhCRT and PI}

The purified rEhCRT from E. coli appeared as a single band at $60 \mathrm{kDa}$ on $12 \%$ SDS-PAGE after bromophenol blue staining (Figure 1A). The results indicate that $\mathrm{rEhCRT}$ was a good immunogenic factor, and this was previously confirmed by the antibody production in animal models (rabbits and mice) (González et al., 2011). The rEhCRT conserved its reactivity when it was tested as an antigen using serum from ALA patients in ELISA assays (Table 1) and in Western blots, and no reactivity was observed with anti-E. coli LPS antibody (Figure 1B).

The role of rEhCRT as a costimulatory factor in the proliferation of PBMC was verified when cellular proliferation was measured by 3-[4,5-dimethylthiazol-2-yl]-2,5 diphenyl tetrazolium bromide (MTT) assays, comparing the stimulatory capacity of rEhCRT vs. the mitogen activity of ConA. The differences in the PI values were not statistically significant for any time point when comparing between the NEG and ALA groups (AP-ALA, R-ALA) (Figure 2). However, at $72 \mathrm{~h}$, we can see the highest PI (Figure 3) and the PI $\geq 1$ is considered as positive proliferation (Verma et al., 2010).

\section{Detection of Cytokines in the Supernatants of PBMC Cultures}

The concentrations of cytokines in PBMC samples obtained for sera from the ALA and NEG groups and treated with EhCRT were measured through ELISA only at $72 \mathrm{~h}$ (Figure 4). The

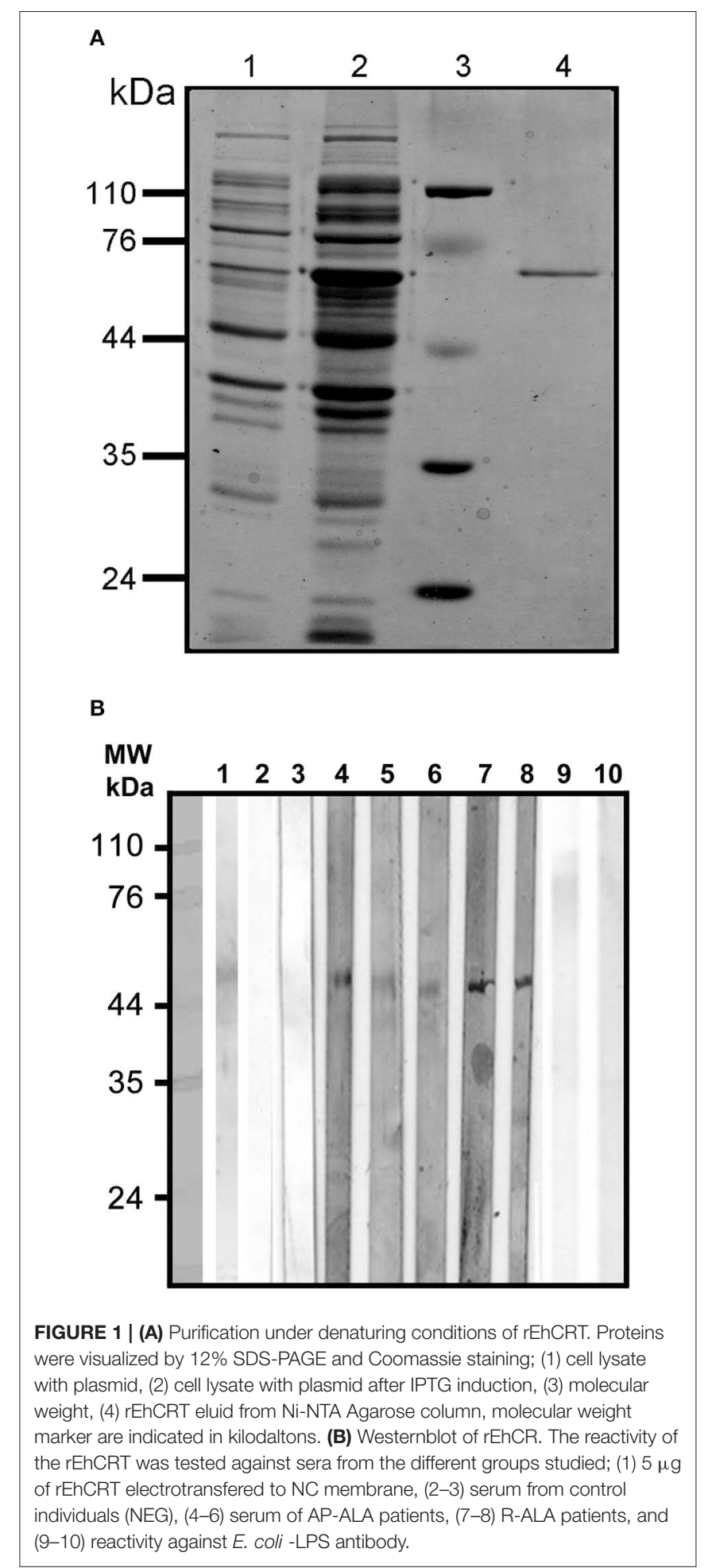

pattern of pro-inflammatory cytokines from the AP-ALA and R-ALA groups were compared to the NEG group, who did not produce the mentioned cytokines in the absenceof EhCRT.

The trend of the data agrees with an overexpression of all interleukins analyzed in all groups. 


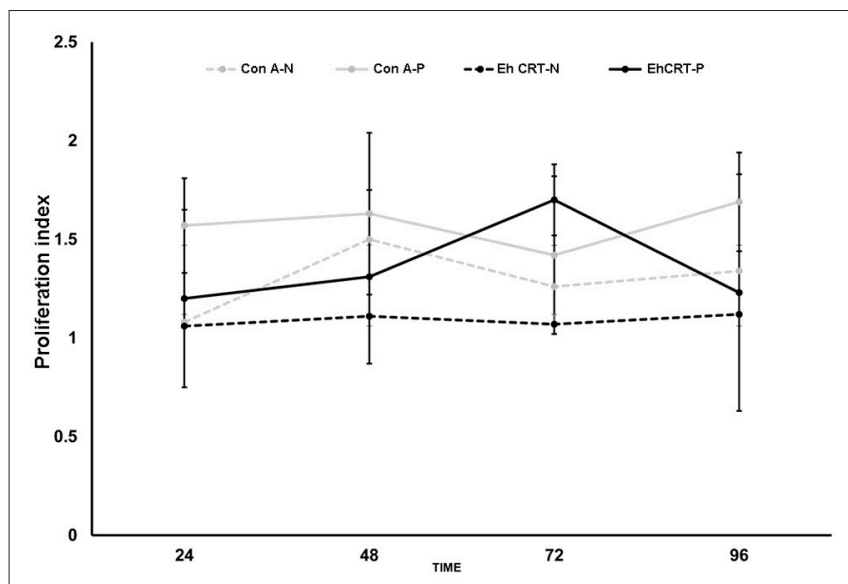

FIGURE 2 | Proliferation Index at different times, of PBMC cells obtained from control subjects (NEG) and ALA patients stimulated with rEhCRT or Con A.

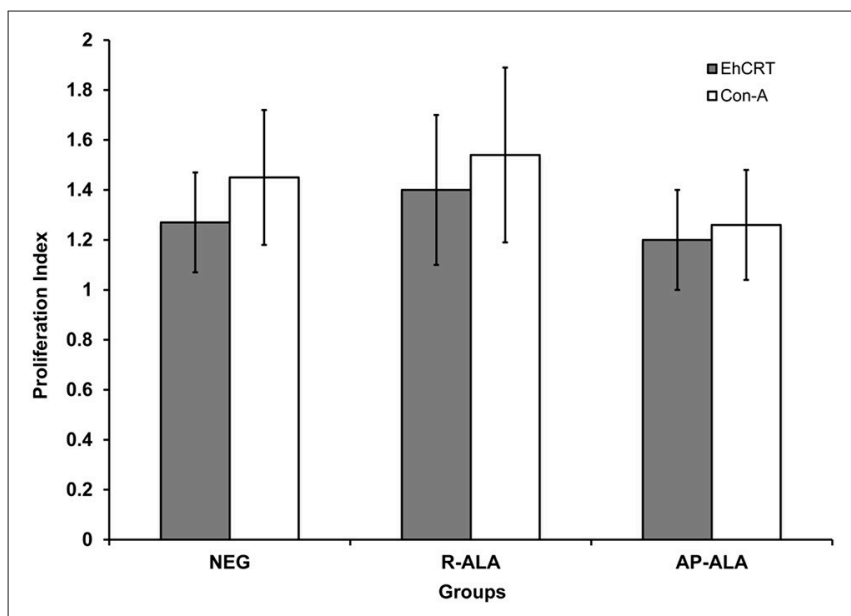

FIGURE 3 | Proliferation Index at $72 \mathrm{~h}$ post incubation of PBMC cells obtained from control subjects (NEG), AP-ALA or R-ALA patients with rEhCRT or Con A. $\mathrm{PI} \geq 1$ is considered as positive stimulus.

However, in the ALA groups, the overexpression was, in general, larger than in the negative group, and significant differences were measured in the expression of the following interleukins; IL-2, IL-5, IL-6, IL-10, IL-17, IFN $\gamma$, TNF $\alpha$, GCSF, and TGF $\beta 1(p=0.0035,0.0023,0.019,0.006,0.0076,0.0049$, $0.0039,0.0035,0.0059$, respectively).

The comparisons of differential expressions of the interleukins between groups of AP-ALA and R-ALA are shown in Figure 4. We found differences in the overexpression of interleukins IL-2, IL-5, IL-6, IL-10, GCSF, and TGF $\beta 1 \quad(p=0.0067$, $0.026,0.0045,0.0016,0.0051,0.0047$, respectively), which were higher in the AP-ALA group. In the R-ALA group, we observed higher overexpression of the interleukins IL-17, INF $\gamma$, and TNF $\alpha$; however, statistically significant differences were detected only for INF $\gamma(p=0.73,0.0014$, 0.096).

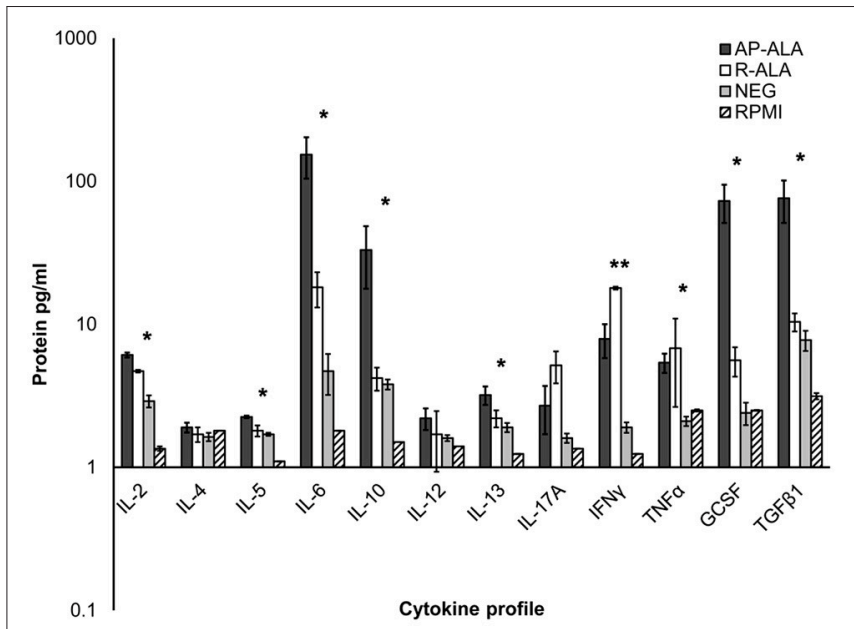

FIGURE 4 | Cytokine profile on PBMC cells stimulated with rEhCRT. The data shows the concentration of cytokines, obtained in the supernatant of PBMC cells of different studied groups (NEG, AP-ALA, and R-ALA) after $72 \mathrm{~h}$ of incubation and stimulated with rEhCRT or without stimulus (RPMI). The concentration of interleukins is expressed as $\mathrm{pg} / \mathrm{ml}$ in a logarithmic scale. ${ }^{*} p \leq$ 0.005 , when compared groups of individuals (NEG) against the group of patients (ALA), ${ }^{* *}$ when compared AP-ALA against R-ALA groups.

\section{DISCUSSION}

The aim of this study was to examine the proliferation of PBMCs obtained from the blood of patients with AP-ALA and R-ALA stimulated in vitro with rEhCRT or ConA and determine the cytokine profiles induced in the different groups. The responses of the PBMC show that EhCRT is one of the many immunogenic proteins in E. histolytica that can induce activation and proliferation of PBMCs similarly to ConA. These results reinforce our previous observations that the EhCRT is highly immunogenic in humans, mice, and rabbits (González et al., 2002, 2011).

When comparing the cytokine profiles between the AP-ALA and R-ALA groups in contrast with negative or PBMC without stimulus, it is clear that EhCRT functions as a specific antigenic costimulator, inducing a different pattern of cytokines between different groups. This stimulatory action was specific because no reactivity with $E$. coli -LPS antibody was detected for the recombinant protein EhCRT (Figure 1A).

The expression levels of IL-2, IL-5, IL-6, IL-10, GCSF, and TGF $\beta 1$ were increased in patients with AP-ALA, while the expression levels of IL-17, INF $\gamma$, and TNF $\alpha$ were mainly upregulated in the R-ALA group. Nevertheless, there were statistically significant differences only for INF $\gamma$.

E. histolytica has different proteins that modulate the host immune response. The Gal/GalNAc-lectin induces the T cells to production of IL-2 and INFy (Schain et al., 1992), whereas in macrophages, the amebicide activity of Gal/GalNAc-lectin induces the production of TNF $\alpha$ (Seguin et al., 1997). In dendritic cells, Gal/GalNAc-lectin favors a Th1 response in addition to inducing the production of major histocompatibility complex 
(MHC) class II molecules, and the costimulatory molecules CD80, CD86, and CD40 (Ivory and Chadde, 2007).

Another protein called monocyte locomotion inhibitory factor (MLIF) is produced by E. histolytica in axenic cultures that induces the production of pro-inflammatory cytokines (IL-1 $\beta$, IL-2, IL-5, IL-6, IFN- $\gamma$ ) and anti-inflammatory cytokines such as IL-10, as well as the low expression of chemokines CCL1, CCL4, and the receptor CCR1 in human monocytes (Rico et al., 2003; Utrera-Barillas et al., 2003).

In the group of patients with AP-ALA, the cytokines IL2, IL5, Il6, IL17A, IFN $\gamma$, and TNF $\alpha$ displayed an increase in their concentration and demonstrated that the immune response had a pro-inflammatory profile. This response has been observed in stimulation assays using Gal/GalNAc-lectin in intestinal cell cultures (Sharma et al., 2005) and in other parasitic diseases such as malaria (Vazquez et al., 2015). It is important to highlight the effect on IL2, IL6, IL-17, IFN $\gamma$, and TNF $\alpha$, whose levels were the highest and overexpressed in comparison with the profile observed in the negative group.

Guo et al. (2011), demonstrated in an Entamoeba histolytica vaccination model that IL-17 provides protection to mice vaccinated with the recombinant LecA fragment of the $\mathrm{Gal} / \mathrm{GalNAc}$-lectin. Interestingly, the major source of IL-17 in these mice was the CD8 $\mathrm{T}$ cells, whereas $\mathrm{CD} 4 \mathrm{~T}$ cells express elevated levels of IFN- $\gamma$. The authors suggest that IL-17 may enhance the protective functions of Th1 immune response.

These results lead us to propose that treatment of PBMC in culture with EhCRT favors the production of IFN $\gamma$ and increases the production of IL-17A, thus directing the cellular immune response to a Th1 Profile, in PMBCs obtained from R-ALA.

Our results agree with those published by Ghadirian and Denis (1992), who showed that IFN $\gamma$ could activate mouse peritoneal macrophages, which, in turn, were able to eradicate the E. histolytica trophozoites from colon tissue in vitro. Studies in animal models (Seydel et al., 2000) and human infections (Haque et al., 2007) have established that amoeba-specific IFN- $\gamma$ production is critically involved in the clearance of infection and in host protection. In addition, Meza et al. (2014) demonstrated that virgin T-cell differentiation into Th17 cells producing IL17 occurred after the direct stimulation with other cytokines such as TGF $\beta$, IL- 6 , and IL-1 in a murine model of infection with Trypanosoma cruzi. Moraes et al. (2015) reported that mononuclear cells collected from healthy individuals incubated with E. histolytica in culture induced the production of IFN $\gamma$ and TGF $\beta$, and that both had a beneficial effect on the modulation of the activity of these cells. Our results agree with data of Moraes regarding the effects on IFN $\gamma$. These cytokines are important in the control E. histolytica infection.

The R-ALA group showed an increase in the concentration of the cytokines IL-10 and TGF $\beta$, which agrees with results of Bansal et al. (2005). These authors mention that, in addition to the production of these cytokines and the increased production of IL-4, a suppressive immune response was also induced in patients infected with E. histolytica, which, in turn, favored a symptomatic infection. The symptomatic group in Bansal et al. differs with our R-ALA group because the latter had no symptoms and they were all ALA with negative microscopic examinations and a resolved
E. histolytica infection. In our opinion, this immunosuppressive effect is due to the direct stimulation of the PBMC by EhCRT, and through autocrine, paracrine, and pleiotropic effects on cytokine production, which favored the increase in other types of cytokines such as IL-5, IL-6, and IL-13 capable of generating a Th2 immune response.

In addition, we found that the increased cytokine IL-10 in the AP-ALA group in our study was in contrast with the results reported by Bansal. These results suggest that IL-10 is an immunomodulator resulting in proinflammatory cytokine profiles that could promote immunosuppression in the R-ALA individuals. The effect attributed to IL-10 differs in other parasitic diseases such as Leishmania donovani (Andreani et al., 2015), Trypanosoma cruzi (Longhi et al., 2014), and Giardia duodenalis (Babaei et al., 2016). In these reports, a decrease in IL-10 was observed that favored the spread of parasites into the hepatic tissue.

On the contrary, the IL-10 level was increased in dysenteric and ALA patients (Utrera-Barillas et al., 2003; Bansal et al., 2005). These studies indicate that invasion of the colon and liver by E. histolytica elicits an anti-inflammatory immune response and may successfully suppress immune reaction to the amebae.

In summary, the ameba needs to balance IL-10 and the proinflammatory cytokine to allow establishment of infection. In contrast, peritoneal monocytes and macrophages exposed to lipopeptidophosphoglycan (LPPG) secreted TNF- $\alpha$, IL-6, IL-8, IL-12, and IL-10 via TLR2 (Maldonaldo-Bernal et al., 2005). Thus, LPPG-driven signaling may activate a negative feedback loop that attenuates inflammatory responses.

Host protective immunity involves participation of both humoral and cellular responses; however, the mechanism involved in the immune evasion of E. histolytica is not clear. One of these mechanisms could be associated with the ability of parasites to modulate cytokine expression in the inflammatory process, which is initiated by expression of proinflammatory cytokines. E. histolytica infections induce a state of transient suppression of cell-mediated immunity in early stages of inflammation in amebic hepatic abscess, and a complex signaling system of cytokines is triggered by pathogen invasion (Eckmann et al., 1993; Romagnani, 2000).

\section{CONCLUSIONS}

The data obtained in this study confirmed that the EhCRT behaves like an amebic immunogenic protein for humans and suggest that the EhCRT participates in the specific stimulation of immune cells.

Our results suggest that the $\mathrm{rEhCRT}$ can stimulate human PBMC proliferation independently of the presence of E. histolytica trophozoites, acting as a specific costimulator of the immune response like that induced by ConA. In addition, these results underline EhCRT as a parasitic factor that can modulate the immune response, from the stimulation of proinflammatory cytokines to the immunosuppressive effects, depending on the progression of the ALA, thus inducing the development of a Th2 cytokine profile in the acute phase of 
disease and a Th1 profile once the individuals had resolved the ALA.

The functions of EhCRT and its role in the pathogenesis of ALA need further research, particularly on the interaction with the cells of immune system and the induction of chemokines and cytokines regulators that hopefully will allow a better understanding of the pathogenesis of ALA.

\section{AUTHOR CONTRIBUTIONS}

CX conceptualized the manuscript. EG, MN-R, UM. PM, OP-R, $\mathrm{EH}, \mathrm{LR}$, and AS curated the data. CX, EG, MN-R, PM, OP-R, $\mathrm{EH}, \mathrm{LR}$, and AS performed the formal analysis. CX acquired the funding. EG, MN-R, and PM performed the investigation. MN-R, PM, AS, and UM provided the resources. EG, OP-R, and EH performed the software analysis. CX, EG, MN-R, and PM provided the supervision. CX, EG, MN-R, and PM executed the validation. $\mathrm{CX}, \mathrm{EG}, \mathrm{MN}-\mathrm{R}$, and $\mathrm{PM}$ wrote the original draft of the manuscript. CX, EG, MN-R, PM, OP-R, EH, UM, and AS wrote, reviewed and edited the manuscript.

\section{REFERENCES}

Andreani, G., Ouellet, M., Menasria, R., Gomez, A. M., Barat, T., and Tremblay, M. J. (2015). Leishmania infantum amastigotes trigger a subpopulation of human B cells with an immunoregulatory phenotype. PLoS Negl. Trop. Dis. 9:e0003543. doi: 10.1371/journal.pntd.0003543

Babaei, Z., Malihi, N., Zia-Ali, N., Sharifi, I., Mahommadi, M. A., Kagnoff, M. F. et al. (2016). Adaptative immune response in symptomatic and asymptomatic enteric protozoal infection: evidence for a determining role of parasite genetic heterogeneity in host immunity to human giardiasis. Microbes Infect. 18, 687-695. doi: 10.1016/j.micinf.2016.06.009

Bansal, D., Sehgal, R., Chawla, Y., Malla, N., and Mahajan, R. C. (2005). Cytokine mRNA expressions in symptomatic vs. Asymptomatic amoebiasis patients. Parasite Immunol. 27, 37-43. doi: 10.1111/j.1365-3024.2005.00739.x

Blazquez, S., Rigothier, M. C., Huerre, M., and Guillen, N. (2007). Initiation of inflammation and cell death during liver abscess formation by Entamoeba histolytica depends on activity of the galactose/ $\mathrm{N}$-acetyl-D-galactosamine lectin. Int. J. Parasitol. 37, 425-433. doi: 10.1016/j.ijpara.2006.10.008

Chadee, K., Meerovitch, E., and Moreau, F. (1985). In vitro and in vivo interaction between trophozoites of Entamoeba histolytica and gerbil lymphoid cells. Infect. Immun. 49, 828-832.

Eckmann, L., Jung, H. C., Schurer-Maly, C., Panja, A., Morzycka- Wroblewska, E., and Kagnoff, M. F. (1993). Differential cytokine expression of interleukin 8. Gastroenterology 105, 1689-1697. doi: 10.1016/0016-5085(93)91064-O

Eckmann, L., Reed, S. L., Smith, J. R., and Kagnoff, M. F. (1995). Entamoeba histolytica trophozoites induce an inflammatory cytokine response by cultured human cells through the paracrine action of cytolytically released interleukin1 $\alpha$. J. Clin. Invest. 96, 1269-1719. doi: 10.1172/JCI118161

El-Gengehi, N., El-Ridi, R., Tawab, N. A., El-Demellawy, M., and Mangold, B. L. (2000). A Schistosoma mansoni $62 \mathrm{kDa}$ band is identified as an irradiated vaccine T-cell antigen and characterized as calreticulin. J. Parasitol. 86, 993-1000. doi: 10.1645/0022-3395(2000)086[0993:ASMKBI]2.0.CO;2

Ferreira, V., Molina, M. C., Valck, C., Rojas, A., Aguilar, L., Ramírez, G., et al. (2004). Role of calreticulin from parasites in its interaction with vertebrate hosts. Mol. Immunol. 40, 1279-1291. doi: 10.1016/j.molimm.2003. 11.018

Garcia-Nieto, R. M., Rico-Mata, R., Arias-Negrete, S., and Avila, E. E. (2008). Degradation of human secretory IgA1 and IgA2 by Entamoeba histolytica surface-associated proteolytic activity. Parasitol. Int. 57, 1417-1423. doi: $10.1016 /$ j.parint.2008.04.013

\section{FUNDING}

We are grateful for the support provided by the following institutions: Grants: IN218214 from PAPIIT (DGAPA), Universidad Nacional Autónoma de México (UNAM), and 272601 from the Consejo Nacional de Ciencia y Tecniología (CONACyT).

\section{ACKNOWLEDGMENTS}

We thank Mario Nequiz-Avendaño for the axenic culture of E. histolytica (HM1:IMSS), and Marco Gudiño Zayas, for art design. LR-V, a doctoral student from the Programa de Doctorado en Ciencias Biomédicas, Universidad Nacional Autónoma de México (UNAM), is grateful for having received fellowship 239901 from CONACYT. We also appreciate the technical assistance of Martha Elena Zaragoza and Ma. de los Angeles Padilla, and the secretarial assistance of Mrs. Ma. Elena Ortiz.

Ghadirian, E., and Denis, M. (1992). In vivo activation of macrophages by IFNgamma to kill Entamoeba histolytica trophozoites in vitro. Parasite Immunol. 14, 397-404. doi: 10.1111/j.1365-3024.1992.tb00014.x

González, E., García de León, M. C., Meza, I., Ocadiz-Delgado, R., Gariglio, P., Silva-Olivares, A., et al. (2011). Entamoeba histolytica Calreticulin: an endoplasmic reticulum protein expressed by trophozoites into experimentally induced amoebic liver abscess. Parasitol. Res. 108, 439-449. doi: 10.1007/s00436-010-2085-6

González, E., Rico, G., Mendoza, G., Ramos, F., García, G., Morán, P., et al. (2002). Calreticulin-like molecule in trophozoites of Entamoeba histolytica HM1: IMSS. Am. J. Trop. Med. Hyg. 67, 636-639. doi: 10.4269/ajtmh.2002. 67.636

Guo, X., Roberts, M. R., Becker, S. M., Podd, B., Zhang, Y., Chua, S. C., et al. (2011). Leptin signalling in intestinal epithelium mediates resistance to enteric infection by Entamoeba histolytica. Mucosal. Immunol. 4, 294-303. doi: $10.1038 / \mathrm{mi} .2010 .76$

Haque, R., Huston, C. D., Hughes, M., Houpt, E., and Petri, W. A. Jr. (2003). Current concepts: amebiasis. N. Engl. J. Med. 348, 1565-1573. doi: 10.1056/NEJMra022710

Haque, R., Mondal, D., Shu, J., Roy, S., Kabir, M., Davis, A. N., et al. (2007). Correlation of interferon-gamma production by peripheral blood mononuclear cells with childhood malnutrition and susceptibility to amebiasis. Am. J. Trop. Med. Hyg. 76, 340-344. doi: 10.4269/ajtmh.2007.76.340

Ivory, C. P. A., and Chadde, K. (2007). Activation of dendritic cells by the Gallectin of Entamoeba histolytica drives Th1 responses in vitro and in vivo. Eur. J. Immunol. 37, 385-394. doi: 10.1002/eji.200636476

Jaworski, D. C., Simmen, F. A., Lamoreaux, W., Coons, L. B., Muller, M. T., and Needham, G. R. (1995). A secreted calreticulin protein in ixoidid tick (Amblyomma americanum) saliva. J. Insect Physiol. 41, 369-375. doi: 10.1016/0022-1910(94)00107-R

Joshi, M., Pogue, G. P., Duncan, R. C., Lee, N. S., Singh, N. K., Atreya, C. D., et al. (1996). Isolation and characterization of Leishmania donovani calreticulin gene and its conservation of the RNA binding activity. Mol. Biochem. Parasitol. 81, 53-64. doi: 10.1016/0166-6851(96)02676-X

Khalife, J., Liu, J. L., Pierce, R., Porchet, E., Godin, C., and Capron, A. (1994). Characterization and localization of Schistosoma mansoni calreticulin Sm5. Parasitol. 108, 527-532. doi: 10.1017/S0031182000077398

Loftus, B., Anderson, I., Davies, R., Alsmark, U. C., Samuelson, J., Amedeo, P., et al. (2005). The genome of the protist parasite Entamoeba histolytica. Nature 433, 865-868. doi: 10.1038/nature03291 
Longhi, S. A., Atienza, A., Perez Prados, G., Buying, A., Balouz, V., Buscaglia, C. A., et al. (2014). Cytokine production but lac of proliferation in peripheral blood mononuclear cells from chronic Chagas's disease cardiomyopathy patients in response to T. cruzi ribosomal P proteins. PLoS Negl. Trop. Dis. 8:e2906. doi: 10.1371/journal.pntd.0002906

Lotter, H., Helk, E., Bernin, H., Jacobs, T., Prehn, C., Adamski, J., et al. (2013). Testosterone increases susceptibility to amebic liver abscess in mice and mediates inhibition of IFN $\gamma$ secretion in natural killer T cells. PLOS ONE 8:e55694. doi: 10.1371/journal.pone.0055694

Maldonaldo-Bernal, C., Kirschning, C. J., Rosenstein, Y., Rocha, L. M., Rios-Sarabia, N., Espinosa-Cantellano, M., et al. (2005). The innate immune response to Entamoeba histolytica lipopeptido phosphoglycan is mediated by toll-like receptors 2 and 4. Parasite Immunol. 27, 127-137. doi: 10.1111/j.1365-3024.2005.00754.x

Marcelain, K. A., Colombo, M. C., Molina, L., Ferreira, M., Lorca, J. C., Aguillón, J. C., et al. (2000). Development of an immunoenzymatic assay for the detection of human antibodies against Tripanosoma cruzi calreticulin, an immunodominant antigen. Acta Tropica 75, 291-300. doi: 10.1016/S0001-706X(00)00062-0

Meza, S. K., Kaneshima, E., de Oliveira, S., Gabriel, M., de Araujo, S., Gomes, M. L., et al. (2014). Comparative pathogenicity in Swiss mice of Trypanosoma cruzi IV from northern Brazil and Trypanosoma cruzi II from southern Brazil. Exp. Parasitol. 146, 34-42. doi: 10.1016/j.exppara.2014. 08.014

Moraes, L. C., Franca, E. L., Pessoa, R. S., Fagundes, D. L., Hernandes, M. G., Ribeiro, V. P., et al. (2015). The effect of IFN- $\gamma$ and TGF- $\beta$ in the functional activity of mononuclear cells in the presence of Entamoeba histolytica. Parasit Vec. 8:413. doi: 10.1186/s13071-015-1028-6

Morán, P., Gómez, A., Valadez, A., Ramos, F., and González, E. (2007). “Amebic and pyogenic liver abscess: importance of differential diagnosis in endemic areas of amebiasis," in 5th European Congress on Tropical Medicine and International Health, ed M. J. Boeree (Amsterdam), 57-64.

Nakashi, H. L., Pogue, G. P., Duncan, R. C., Joshi, M., Atreya, C. D., Lee, N. S., et al. (1998). Implications of calreticulin function in parasite biology. Parasitol. Tdy 14, 157-160. doi: 10.1016/S0169-4758(97)01180-0

Naresha, S., Suryawanshi, A., Agarwal, M., Sing, B. P., and Joshi, P. (2009). Mapping the complement $\mathrm{Clq}$ binding site in Haemonchus contortus calreticulin. Mol. Biochem. Parasitol. 166, 42-46. doi: 10.1016/j.molbiopara.2009.02.007

Rico, G., Leandro, E., Rojas, S., Jiménez, J. A., and Kretschmer, R. (2003). The monocyte locomotion inhibitory factor produced by Entamoeba histolytica inhibits induced nitric oxide production in human leukocytes. Parasitol. Res. 90, 264-267. doi: 10.1007/s00436-002-0780-7

Rokeach, L. A., Zimmerman, P. A., and Unnasch, T. R. (1994). Epitopes of Onchocerca volvulus $\mathrm{RAL}-1$ antigen a member of the calreticulin family of proteins recognized by sera from patients with onchocerciasis. Infect. Immun. 62, 3696-3704. PMCID:PMC303020

Romagnani, S. (2000). T-cell subsets (Th1 versus Th2). Ann. Allergy Asthma Immunol. 85, 9-18. doi: 10.1016/S1081-1206(10)62426-X

Schain, D. C., Salata, R. A., and Raydin, J. I. (1992). Human T-lymphocyte proliferation lymphokine production and amebicidal activity elicited by the galactose-inhibitable adherence protein of Entamoeba histolytica. Infect. Immun. 60, 2143-2146.
Seguin, R., Mann, B. J., Keller, K., and Chadee, K. (1997). The tumor necrosis factor alpha-stimulating region of galactoses-inhibitable lectin of Entamoeba histolytica activates gamma interferon-primed macrophages for amebicidal activity mediated by nitric oxide. Infect. Immun. 65, 2522-2527.

Seydel, K. B., Smith, S. J., and Stanley, S. L. Jr. (2000). Innate immunity to amebic liver abscess is dependent on gamma interferon and nitric oxide in a murine model of disease. Infect. Immun. 68, 400-102. doi: 10.1128/IAI.68.1.400-402.2000

Sharma, M., Vohra, H., and Bhasin, D. (2005). Enhanced pro-inflammatory chemokine/cytokine response triggered by pathogenic Entamoeba histolytica: basis of invasive disease. Parasitology 131, 783-796. doi: $10.1017 /$ S0031182005008541

Suchitra, S., and Joshi, P. (2005). Characterization of Haemonchus contortus calreticulin suggests its role in feeding and immune evasion by the parasite. Biochim. Biophys. Acta 1722, 293-303. doi: 10.1016/j.bbagen.2004.12.020

Talvani, A., Rocha, M. O., Ribeiro, A. L., Correa-Oliveira, R., and Teixeira, M. M. (2004). Chemokine receptor expression on the surface of peripheral blood mononuclear cells in chagas disease. J. Infect. Dis. 189, 214-220. doi: $10.1086 / 380803$

Utrera-Barillas, D., Velazquez, J., Enciso, A., Cruz, S., Rico, G., Curiel-Quesada, E., et al. (2003). An anti-inflammatory oligopeptide produced by Entamoeba histolytica down-regulates the expression of pro-inflammatory chemokines. Parasit. Immunol. 25, 475-482. doi: 10.1111/j.1365-3024.2003.00657.x

Vaithilingam, A., Teixeira, J. E., Miller, P. J., Heron, B. T., and Huston, C. D. (2012). Entamoeba histolytica cell surface calreticulin binds human C1q and functions in amebic phagocytosis of host cells. Infect. Immun. 80, 2008-2018. doi: 10.1128/IAI.06287-11

Vazquez, B. P., Vazquez, T. P., Miguel, C. B., Rodríguez, W. F., and Méndez, M. T. (2015). Inflammatory responses and intestinal injury development during acute Trypanosoma cruzi infection are associated with the parasite load. Parasit. Vec. 8:206. doi: 10.1186/s13071-015-0811-8

Verma, A., Prasad, K. N., Singh, A. K., Nyati, K. K., Gupta, R. K., and Paliwal, V. M. (2010). Evaluation of the MTT lymphocyte proliferation assay for the diagnosis of neurocysticercosis. J. Microbiol. Methods 81, 175-178. doi: 10.1016/j.mimet.2010.03.001

Ximénez, C., González, E., Nieves, M. E., Silva-Olivares, A., Shibayama, M., Galindo-Gómez, S., et al. (2014). Entamoeba histolytica and E. dispar Calreticulin: inhibition of classical complement pathway and differences in the level of expression in amoebic liver abscess. Biomed. Res. Int. 2014:127453. doi: $10.1155 / 2014 / 127453$

Conflict of Interest Statement: The authors declare that the research was conducted in the absence of any commercial or financial relationships that could be construed as a potential conflict of interest.

Copyright (๑) 2018 Gonzalez Rivas, Ximenez, Nieves-Ramirez, Moran Silva, PartidaRodríguez, Hernandez, Rojas Velázquez, Serrano Vázquez and Magaña Nuñez. This is an open-access article distributed under the terms of the Creative Commons Attribution License (CC BY). The use, distribution or reproduction in other forums is permitted, provided the original author(s) and the copyright owner(s) are credited and that the original publication in this journal is cited, in accordance with accepted academic practice. No use, distribution or reproduction is permitted which does not comply with these terms. 